\title{
Quantum effects in large molecule collisional energy transfer?
}

\author{
Beatriz M. Toselli and John R. Barker ${ }^{1}$ \\ Department of Atmospheric, Oceanic, and Space Sciences, Space Physics Research Laboratory. The University of Michigan, \\ Ann Arbor, MI 48109-2143, USA
}

Received 6 August 1990; in final form 22 August 1990

\begin{abstract}
Recently, Gilbert and Zare proposed that dynamical quantum effects might explain the poor performance of classical trajectory calculations in simulating the vibrational deactivation of excited azulene by the lighter noble gases. They proposed an experimental test: a comparison of ${ }^{3} \mathrm{He}$ and ${ }^{4} \mathrm{He}$ deactivation of azulene. In this Letter, the collisional deactivation of benzene, toluene and toluene- $d_{8}$ by ${ }^{3} \mathrm{He}$ and ${ }^{4} \mathrm{He}$ has been investigated by infrared fluorescence to assess the importance of dynamical quantum effects. The results show that the proposed dynamical quantum effect is not important for these systems over the range of vibrational energies from $\approx 8000$ to $\approx 35000 \mathrm{~cm}^{-1}$.
\end{abstract}

\section{Introduction}

The collisional relaxation of highly excited polyatomic molecules is an important but not well understood topic in gas-phase chemical reactions, and it has been attacked by a number of experimental and theoretical approaches. Traditionally, energy transfer was investigated in unimolecular reaction systems [1-3], but recently the so-called "direct methods" that measure physical rather than chemical properties have provided a more detailed picture about the average energy transferred per collision, $\langle\Delta E\rangle$, in the vibrational deactivation of large molecules in the electronic ground state. In our laboratory we have been using both the time-resolved infrared fluorescence (IRF) and time-dependent thermal lensing techniques to measure energy transfer. With the IRF technique we have measured the collisional deactivation of azulene [4,5], 1,1,2-trifluoroethane [6], benzene [7], and some of its derivatives $[8,9]$; with the TDTL technique we have measured the collisional deactivation of highly excited $\mathrm{NO}_{2}$ after laser irradiation at $460 \mathrm{~nm}$ [10]. Troe and coworkers have used time-resolved ultraviolet absorption to study energy transfer in several large polyatomic molecules $[11,12], \mathrm{CF}_{3} \mathrm{I}[13]$ and the

\footnotetext{
I And Department of Chemistry.
}

triatomics $\mathrm{SO}_{2}$ [14] and $\mathrm{CS}_{2}$ [15]. Techniques developed by Luther and coworkers [16] and by Weisman and coworkers [17] promise to provide information that is even more detailed.

The main objective of all of these measurements has been to characterize the dependence of $\langle\Delta E\rangle$ on the vibrational energy, temperature, and molecular parameters. The results of these studies show that many factors play a role in the energy transfer process and thus a broad-based theory is needed for an adequate description. The earliest theoretical approaches and their descendents are based on simple models that rely heavily on statistical assumptions $[3,18]$. Recently, several groups have carried out classical trajectory calculations on energy transfer systems, although the validity of this approach is still somewhat uncertain. Schatz and coworkers [19] have done extensive studies of $\mathrm{CS}_{2} /$ inert gas systems and Lim and Gilbert [20] have reported calculations for deactivation of highly vibrationally excited azulene by the noble gases. The results of the azulene study were mixed: good agreement was obtained with experimental data $[4,12]$ for the heavier colliders, but poor agreement for the lighter ones. The discrepancy was about a factor of 5 for the $\mathrm{He}$ /azulene system. Lim and Gilbert proposed that the discrepancies for the lighter colliders may be due to erroneous assumed potential energy parameters, or to a 
possible failure of classical mechanics to simulate quantum systems. They concluded that the more likely culprit was the choice of potential parameters.

Recently, Gilbert and Zare [21] revisited the discrepancy between trajectory calculations and the experiments for light colliders and conjectured that the failure of classical calculations could be due a dynamical quantum effect, if not due to errors in the assumed potential energy function (they also considered, and dismissed, two other possible quantum effects: the effect of the zero-point energy and the quantum propensity rules). The dynamical quantum effect arises from the fact that classical collision cross sections are infinite, while quantum cross sections are finite, due to interferences among partial waves. Gilbert and Zare conjectured that the classical trajectory calculations erroneously include larger impact parameters than would be permitted by a quantum treatment, thus overestimating energy transfer. This effect would be strongest for lighter colliders. They proposed an excellent experimental test: a direct comparison of ${ }^{3} \mathrm{He}$ and ${ }^{4} \mathrm{He}$ collider gases, which have identical potential energy surfaces, but the lighter gas would show a stronger dynamical quantum effect. In this Letter, we report experimental results for this type of experiment applied to benzene, toluene, and toluene- $d_{8}$. Benzene and its derivatives were used in the present study because they are easier to handle experimentally than azulenc and because they are more convenient for possible future theoretical calculations.

\section{Experimental}

Experiments were carried out with both ${ }^{3} \mathrm{He}$ and ${ }^{4} \mathrm{He}$ for benzene, toluene, and toluene- $d_{8}$ using the IRF technique, which has been described elsewhere [4-7]. Basically, a KrF excimer laser (248 nm) irradiated the gas phase species in a $30 \mathrm{~cm}$ long, 4.5 $\mathrm{cm}$ diameter pyrex cell. IRF was viewed through a quartz side window (to monitor the $\mathrm{C}-\mathrm{H}$ emission near $3.3 \mu \mathrm{m}$ ) or through a $\mathrm{CaF}_{2}$ side window (to monitor the $C-D$ emission near $4.5 \mu \mathrm{m}$ ) with a 3 mm diameter $77 \mathrm{~K}$ InSb photovoltaic detector (Infrared Associates) equipped with a matched preamplifier and appropriate interference filters. The experiments were performed under static bulb conditions in mixtures containing $10 \mathrm{mTorr}$ of benzene or its derivatives ("parent" gas) and up to about 800 mTorr of collider $\left({ }^{3} \mathrm{He}\right.$ or $\left.{ }^{4} \mathrm{He}\right)$. The signals were amplified and averaged with a Tektronix AM 502 accoupled amplifier and averaged with a LeCroy 9400 digital oscilloscope for $\approx 5000$ pulses, in order to achieve good $\mathrm{S} / \mathrm{N}$ ratios in each experiment. The signal was further analyzed after transfer to a Macintosh personal computer. The IRF signals were limited by the $\approx 5 \mu$ s rise time of the infrared detector/preamplifier. The laser fluence employed in the present measurements was $\approx 25 \mathrm{~mJ} \mathrm{~cm}{ }^{-2}$, such that approximately $0.5 \%$ of the molecules in the laser beam were excited.

Benzene (Fisher Scientific, ACS grade), toluene (Aldrich) and toluene- $d_{8}$ (Sigma $99+$ atom\% D) were degassed prior to use. The collider gases ${ }^{3} \mathrm{He}$ (Isotec Inc. DRG, 99.8 atom\% ${ }^{3} \mathrm{He}$ ) and ${ }^{4} \mathrm{He}$ (ultra high pure carrier from Air Products) were used without further purification.

\section{Results}

The experimental results consist of IRF decay curves, which were fitted by nonlinear least squares to an empirical function as in ref. [7]:

$$
\left\langle\langle I(t)\rangle=A \exp \left(-k^{1} t+b^{1} t^{2}\right)+B .\right.
$$

The double brackets indicate that the observed IRF corresponds to the bulk average over the population distribution, which evolves with time. The theoretical relationship between IRF emission intensity and vibrational energy $(E)$ has been discussed elsewhere [5] and it was used with vibrational assignments taken from refs. [22-24] for the excited molecules to calculate $I(E)$ "calibration curves". These curves were used to invert the IRF data to give the bulk average energy $\left\langle\langle E(t)\rangle_{\text {inv }}\right.$ and the bulk average energy transferred per collision $\left\langle\langle\Delta E\rangle_{\mathrm{inv}}\right.$, using the procedure described elsewhere [7-9]. The results of this analysis are plots $\left\langle\langle\Delta E\rangle_{\text {inv }}\right.$ as a function of $\left\langle\langle E\rangle_{\text {inv }}\right.$ for each experimental run, which is characterized by a mixing ratio of parent/collider. Note that in the experiments only the product of $\langle\langle\Delta E\rangle\rangle$ and the gas kinetics rate constant is determined and the value of $\langle\langle\Delta E\rangle$ is inferred by assuming Lennard-Jones parameters for the collision partners to calculate $k_{\mathrm{L}}$ 
Table 1

Lennard-Jones rate constants and average energy transferred per collision

\begin{tabular}{|c|c|c|c|c|}
\hline \multirow{2}{*}{$\begin{array}{l}\text { Excited } \\
\text { molecule }\end{array}$} & \multicolumn{2}{|l|}{ Helium-3 } & \multicolumn{2}{|l|}{ Helium-4 } \\
\hline & $\begin{array}{l}\left.10^{10} k_{\mathrm{LJ}}^{\mathrm{a}}\right) \\
\left(\mathrm{cm}^{3} \mathrm{~s}^{-1}\right)\end{array}$ & $\begin{array}{l}-\left\langle\langle\Delta E\rangle_{i \mathbf{i n}}{ }^{b, c\}}\right. \\
\left(\mathbf{c m}^{-1}\right)\end{array}$ & $\begin{array}{l}10^{10} k_{\mathrm{L}^{\mathrm{a}}}{ }^{-1} \\
\left(\mathrm{~cm}^{3} \mathrm{~s}^{-1}\right)\end{array}$ & $\begin{array}{l}-\left\langle\langle\Delta E\rangle_{\mathrm{inv}}^{\mathrm{b}, c)}\right. \\
\left(\mathrm{cm}^{-1}\right)\end{array}$ \\
\hline $\mathrm{C}_{6} \mathrm{H}_{6}$ & 7.10 & $27 \pm 1$ & 6.16 & $24 \pm 1$ \\
\hline $\mathrm{C}_{7} \mathrm{H}_{8}$ & 8.21 & $60 \pm 1$ & 7.14 & $62 \pm 1$ \\
\hline $\mathrm{C}_{7} \mathrm{D}_{8}$ & 8.20 & $53 \pm 2$ & 7.13 & $60 \pm 1$ \\
\hline
\end{tabular}

a) Calculated using Lennard-Jones parameters from refs. [7,11].

b) Uncertainties are $\pm 2 \sigma$ statistical errors; possible systematic errors are not included.

c) Evaluated at $\langle E\rangle_{\mathrm{inv}}=24000 \mathrm{~cm}^{-1}$.

(table 1). The total average amount of energy transferred per collision, $\left\langle\langle\Delta E\rangle_{\text {inv }}\right.$, for each mixture of parent and collider gases was extrapolated to unit collision fraction in order to get $\langle\langle\Delta E\rangle$ for parentcollider gas collisions, uncontaminated by the effects of parent-parent gas collisions. The collision fraction for an experiment is the fraction of collisions due to parent-collider gas interactions:

collision fraction $=\frac{N_{\mathrm{c}} k_{\mathrm{L}}^{\mathrm{c}}}{N_{\mathrm{c}} k_{\mathrm{L}}^{\mathrm{s}}+N_{\mathrm{p}} k \mathrm{P}_{\mathrm{J}}}$

(where $N_{\mathrm{c}}$ and $N_{\mathrm{p}}$ denote concentrations of collider gas and parent, respectively). A typical plot of $\langle\Delta E\rangle$ versus collision fraction is shown in fig. 1 for

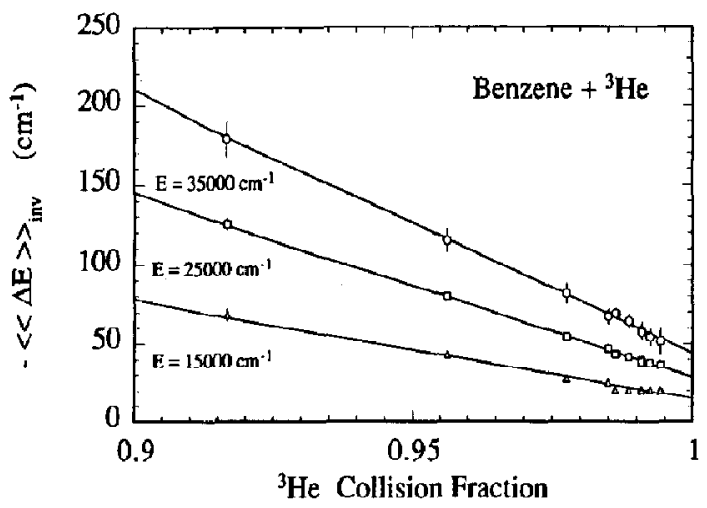

Fig. 1. Average energy transferred per collision versus collision fraction (see the text) for benzene- ${ }^{3} \mathrm{He}$ at three different internal energies. The experimental points at each energy are fit to a quadratic function (solid line) and extrapolation to ${ }^{3} \mathrm{He}$ collision fraction $=1$ yields $\left\langle\langle\Delta E\rangle\right.$ for benzene* ${ }^{*}{ }^{3} \mathrm{He}$ collisions at each energy. benzene- ${ }^{3} \mathrm{He}$. Similar curves were obtained for the other systems at all values of $\left\langle\langle E\rangle_{\text {inv }}\right.$ reported. A complete description of the method of analysis will be published elsewhere [8]. The extrapolated $\left\langle\langle\Delta E\rangle_{\text {inv }}\right.$ for the three systems studied are shown in
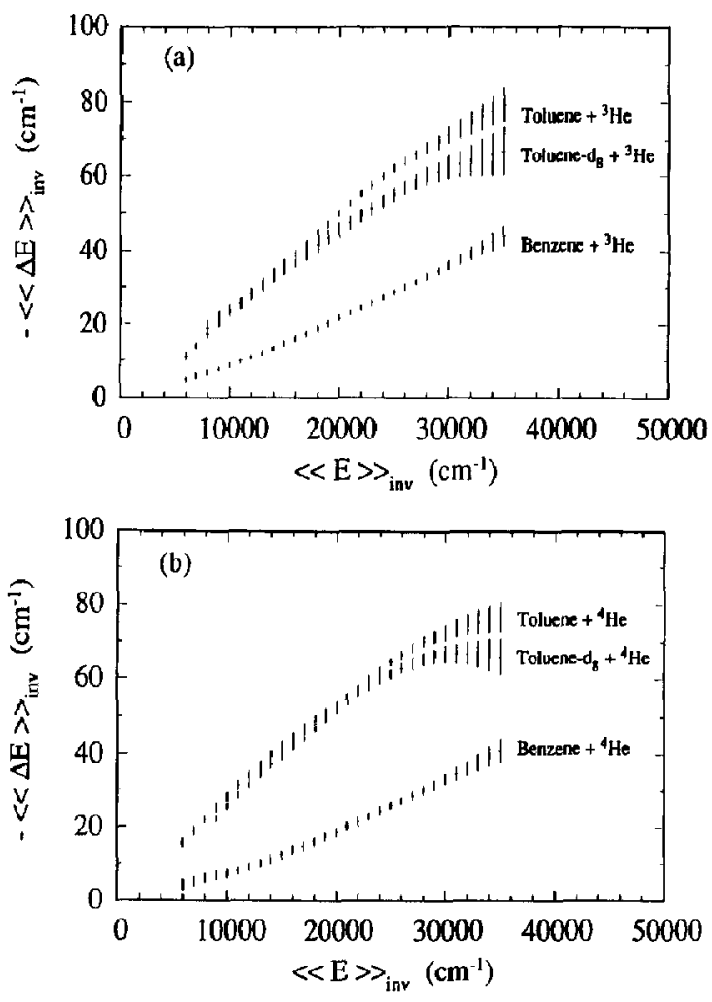

Fig. 2. Average energy transferred per collision versus average vibrational energy (bulk averages). Initial energy $\approx 40800 \mathrm{~cm}^{-1}$. (a) Excited molecules deactivated by ${ }^{3} \mathrm{He}$. (b) Excited molecules deactivated by ${ }^{4} \mathrm{He}$. 
fig. 2, and in table 1 a comparison of $\left\langle\langle\Delta E\rangle_{\text {inv }}\right.$ values at $\left\langle\langle E\rangle_{\text {inv }}=24000 \mathrm{~cm}^{-1}\right.$ is also presented. It is clear that the energy transfer parameters are very similar for both helium isotopes. These experimental results show that the conjectured dynamical quantum effect is not dominating the energy transfer in these systems.

\section{Discussion}

As was mentioned above, the results of Lim and Gilbert for the deactivation of excited azulene by ${ }^{4} \mathrm{He}$ and $\mathrm{Ne}$ are in considerable disagreement with the experimental data. To explain the discrepancies Gilbert and Zare [21] suggested that the poor performance of the classical trajectory calculations could be due either to the dynamical quantum effect described above, or to the parameterization of the potential energy surface. Total collision frequencies calculated using the method of Durant and Kaufman [25] (based on the quantum mechanical collision cross sections) and the Lennard-Jones parameters from Lim and Gilbert [20] are about twice as large as the Lennard-Jones collision frequencies for ${ }^{4} \mathrm{He} /$ azulene collisions. Crudely, this means that the maximum impact parameter for Lennard-Jones collisions is about $30 \%$ smaller than the maximum quantum impact parameter. This result indicates that the limit imposed by the finite nature of the quantum cross section is probably not important in the classical calculation, because the classical trajectory results had virtually no contribution from impact parameters as large as the maximum quantum value, according to fig. 1 in ref. [21]. In view of the results obtained in the present work, the potential energy parameters in the trajectory calculations should be re-examined.

Although the conjectured dynamical quantum effect does not appear to dominate energy transfer in the systems studied, the poor performance of the classical trajectory calculations for light colliders may still be due to quantum effects, at least in part. In the classical trajectory calculations, the zero-point energy ( $\approx 18000 \mathrm{~cm}^{-1}$ for benzene) is included and it is available for energy transfer, unlike quantum systems [26]. For large molecules with 30-40 vibrational modes at an initial energy of $40000 \mathrm{~cm}^{-1}$, the number of quanta in each mode will be small $(\approx 1$ quantum), on the average, and the possible transfer of zero-point energy in classical calculations is likely to make a significant contribution. If this is the only important difference between classical and quantum energy transfer, classical trajectory calculations will always overestimate energy transfer, at least to some extent. This deficiency may be overcome using methods recently proposed for preserving zero-point energy in classical trajectory calculations [27,28], but tests are needed to determine their validity.

Another possible contribution to the difference between classical trajectory calculations and quantum calculations may arise from the differences between classical and quantum statistics. In a collection of coupled classical harmonic oscillators (as in the Lim and Gilbert model for azulene [20]), equipartition of energy occurs, producing (on the average) equal energy in every vibrational mode, whether high, or low frequency. In a quantum system, however, the energy is not distributed equally, if the energy is low enough for the average occupation number to be low: high frequency modes contain very little disposable energy and most of the energy resides in the low frequency modes, on the average. Thus, in a classical system, too much energy resides in the high frequency modes, on the average. This effect, coupled with the relatively high energy transfer efticiency of impulsive collisions [29] may provide a partial explanation for the mass dependence of the discrepancies between calculations and experiment. Collisions of the higher velocity lighter gases with the higher frequency modes will be more effective and transfer more energy than collisions involving the heavier gases. This effect will be less pronounced for the lower frequency modes. Thus the combination of an overabundance of disposable energy in the high frequency modes coupled with a tendency for more efficient energy transfer between high frequency modes and light gases may explain, in part, why the trajectory calculations give good results for the heavier gases, but produce large discrepancies for the lighter gases.

This conjecture can be tested, at least partially, by a comparison of classical trajectory results for protonated and deuterated excited molecules, e.g. $\mathrm{C}_{7} \mathrm{H}_{8}$ and $\mathrm{C}_{7} \mathrm{D}_{8}$, with experimental data, such as those in fig. 2 and table 1 . The tendency for more efficient energy transfer from high frequency modes by more impulsive collisions with helium will favor larger 
$\langle\Delta E\rangle$ values for $\mathrm{C}_{7} \mathrm{D}_{8}$ than for $\mathrm{C}_{7} \mathrm{H}_{8}$, while the overabundance of disposable energy will be about the same for high frequency modes in both molecules (although there will be a slightly larger overabundance for $\mathrm{C}_{7} \mathrm{H}_{8}$ ). Thus, if this conjecture is correct, the classical trajectory calculations will produce significantly larger $\langle\Delta E\rangle$ values for $\mathrm{C}_{7} \mathrm{D}_{8}$ than for $\mathrm{C}_{7} \mathrm{H}_{8}$, distinctly different from the experimental data in fig. 2 and table 1 , which show only small differences, and in the opposite direction. Moreover, the overabundance of disposable energy in both molecules will be reduced if the methods mentioned above $[27,28]$ are used to preserve the zero-point energy.

\section{Conclusions}

The experimental results on the average amount of energy transferred per collision show little difference between ${ }^{3} \mathrm{He}$ and ${ }^{4} \mathrm{He}$ in deactivation of three different excited molecules, indicating that the dynamical quantum effect proposed by Gilbert and Zare is not dominant in these systems. The reasons for the poor performance of classical trajectory calculations might be found in the assumed potential energy parameters and in the unphysical fact that in the calculations the zero-point energy is available for energy transfer. A possible additional factor may be found in the differences between classical and quantum statistics coupled with the relatively higher energy transfer efficiency for lighter gases; these two factors may combine in classical trajectory calculations to give high frequency modes in the excited molecule a much larger role than they should have in energy transfer.

\section{Acknowledgement}

Thanks go to the Department of Energy, Office of Basic Energy Sciences, for funding this work.

\section{Referencęs}

[1] D.C. Tardy and B.S. Rabinovitch. Chem. Rev. 77 (1977) 369.

[2] M. Quack and J. Troe, Gas kinetics and energy transfer, Vol. 2 (Chem. Soc., London, 1977).

[3] 1. Oref and D.C. Tardy, Chem. Rev, in press.

[4] M.J. Rossi, J.R. Paldziewicz and J.R. Barker, J. Chem. Phys. 78 (1983) 6695 , and references therein;

J.R. Barker, J. Phys. Chem. 88 (1984) 11;
J.R. Barker and R.E. Golden, J. Phys. Chem. 88 (1984) 1012.

[5] J. Shi and J.R. Barker, J. Chem. Phys. 88 (1988) 6219; J. Shi, D. Bernfield and J.R. Barker, J. Chem. Phys. 88 (1988) 6211.

[6] J.M. Zellweger, T.C. Brown and J.R. Barker, J. Chem. Phys. 83 (1985) 6261.

[7] M.L. Yerram, J.D. Brenner, K.D. King and J.R. Barker, J. Phys. Chem., in press.

[8] B.M. Roselli, J.D. Brenner, M.L. Yerram, W.E. Chin, K.D. King and J.R. Barker, to be published.

[9] B.M. Toselli and J.R. Barker, to be published,

[10] B.M. Toselli, T.L. Walunas and J.R. Barker, J. Chem. Phys. 92 (1990) 4793.

[11] H. Hippler, J.Troe and J.Wendelken, J. Chem. Phys. 78 (1983) 6709, 6718.

[12] H. Hippler, L. Lindemann and J. Troe, J.Chem. Phys. 83 (1985) 3906;

H. Hippler, B. Otto and J. Troe, Ber, Bunsenges. Physik. Chem. 93 (1989) 428.

[13] B. Abel, B. Herzog, H. Hippler and J. Troe, J. Chem. Phys. 91 (1989) 900.

[14] M. Heymann, H. Hippler, D. Nahr, H.J. Plach and J. Troe, J. Phys. Chem. 92 (1988) 5507.

[15] J.E. Dove, H. Hippler and J.Troe, J. Chem. Phys. 82 (1985) 1907;

M. Heymann, H. Hippler, H.J. Plach and J. Troe, J.Chem. Phys. 87 (1987) 3867.

[16] H.G. Löhmannsröben and K. Luther, Chem. Phys. Letters 144 (1988) 473;

K. Luther, private communication.

[17] T.J. Bevilacqua, B.K. Andrews, J.E.Stout and R.B. Weisman, J. Chem. Phys. 92 (1990) 4627.

[18] H.W. Schranz and S. Nordholm, Intern. J. Chem. Kinetics 25 (1974) 275.

[19] M. Bruehl and G.C. Schatz, J. Chem. Phys. 89 (1988) 770; J. Phys. Chem. 92 (1988) 7223.

[20] K.F. Lim and R.G. Gilbert, J. Phys. Chem. 94 (1990) 77.

[21] R.G. Gilbert and R.N. Zare, Chem. Phys. Letters 167 (1990) 407.

[22] R.H. Page, R.H. Shen and Y.T. Lee, J. Chem. Phys. 88 (1988) 5362 .

[23] J.A. Draeger, Spectrochim. Acta 41 (1985) 607;

H.D. Rudolph, H. Dreizler, A. Jauschke and P. Wendling, Z. Naturforsch. 22 (1967) 940.

[24] N. Fuson, C. Garrigou-Lagrange and M.L. Josien, Spectrochim. Acta 16 (1960) 106.

[25] J.L. Durant and F. Kaufman, Chem. Phys. Letters 142 (1987) 246 .

[26] M.G. Sceats, J. Chem. Phys. 91 (1989) 6795.

[27] J.M. Bowman, B. Gazdy and Q. Sun, J. Chem. Phys. 91 (1989) 2859.

[28] W.H. Miller, W.L. Hase and C.L. Darling, J. Chem. Phys. 91 (1989) 2863.

[29] R.D. Levine and R.B. Bernstein, Molecular reaction dynamics and chemical reactivity (Oxford Univ. Press, Oxford, 1987). 